\title{
Effects of long-term weekly iron and folic acid supplementation on lower genital tract infection - a double blind, randomised controlled trial in Burkina Faso
}

Loretta Brabin ${ }^{1,15^{*}}$ (D), Stephen A. Roberts ${ }^{2}$, Sabine Gies ${ }^{3,4}$, Andrew Nelson ${ }^{5}$, Salou Diallo ${ }^{6}$, Christopher J. Stewart ${ }^{7}$, Adama Kazienga ${ }^{6}$, Julia Birtles ${ }^{8}$, Sayouba Ouedraogo ${ }^{6}$, Yves Claeys $^{9}$, Halidou Tinto ${ }^{10,11}$, Umberto d'Alessandro ${ }^{6}$, E. Brian Faragher ${ }^{12}$ and Bernard Brabin ${ }^{13,14}$

\begin{abstract}
Background: Provision of routine iron supplements to prevent anaemia could increase the risk for lower genital tract infections as virulence of some pathogens depends on iron availability. This trial in Burkina Faso assessed whether weekly periconceptional iron supplementation increased the risk of lower genital tract infection in young non-pregnant and pregnant women.

Methods: Genital tract infections were assessed within a double blind, controlled, non-inferiority trial of malaria risk among nulliparous women, randomised to receive either iron and folic acid or folic acid alone, weekly, under direct observation for 18 months. Women conceiving during this period entered the pregnancy cohort. End assessment (FIN) for women remaining non-pregnant was at 18 months. For the pregnancy cohort, end assessment was at the first scheduled antenatal visit (ANC1). Infection markers included Nugent scores for abnormal flora and bacterial vaginosis (BV), T. vaginalis PCR, vaginal microbiota, reported signs and symptoms, and antibiotic and anti-fungal prescriptions. Iron biomarkers were assessed at baseline, FIN and ANC1. Analysis compared outcomes by intention to treat and in iron replete/deficient categories.

Results: A total of 1954 women (mean 16.8 years) were followed and 478 (24.5\%) became pregnant. Median supplement adherence was 79\% (IQR 59-90\%). Baseline BV prevalence was 12.3\%. At FIN and ANC1 prevalence was $12.8 \%$ and $7.0 \%$, respectively $(P<0.011)$. T. vaginalis prevalence was $4.9 \%$ at FIN and $12.9 \%$ at ANC1 $(P<0.001)$. BV and $T$. vaginalis prevalence and microbiota profiles did not differ at trial end-points. Iron-supplemented nonpregnant women received more antibiotic treatments for non-genital infections ( $P=0.014$; mainly gastrointestinal infections $(P=0.005)$, anti-fungal treatments for genital infections $(P=0.014)$ and analgesics $(P=0.008)$. Weekly iron did not significantly reduce iron deficiency prevalence. At baseline, iron-deficient women were more likely to have normal vaginal flora $(P=0.016)$.

(Continued on next page)
\end{abstract}

\footnotetext{
* Correspondence: loretta.brabin@manchester.ac.uk

${ }^{1}$ Division of Cancer Sciences, Faculty of Biology, Medicine and Health, School of Medical Sciences, University of Manchester, Manchester Academic Health Science Centre, Manchester, UK

${ }^{15}$ Division of Cancer Sciences, 5th (Research) floor, St Mary's Hospital, Oxford

Road, Manchester M13 9WL, UK

Full list of author information is available at the end of the article
}

(c) The Author(s). 2017 Open Access This article is distributed under the terms of the Creative Commons Attribution 4.0 International License (http://creativecommons.org/licenses/by/4.0/), which permits unrestricted use, distribution, and reproduction in any medium, provided you give appropriate credit to the original author(s) and the source, provide a link to the Creative Commons license, and indicate if changes were made. The Creative Commons Public Domain Dedication waiver (http://creativecommons.org/publicdomain/zero/1.0/) applies to the data made available in this article, unless otherwise stated. 
(Continued from previous page)

Conclusions: Periconceptional weekly iron supplementation of young women did not increase the risk of lower genital tract infections but did increase general morbidity in the non-pregnant cohort. Unabsorbed gut iron due to malaria could induce enteric infections, accounting for the increased administration of antibiotics and antifungals in the iron-supplemented arm. This finding reinforces concerns about routine iron supplementation in highly malarious areas.

Trial registration: Trial registration number NCT01210040. Registered with Clinicaltrials.gov on 27 September 2010

Keywords: Lower genital tract infection, Iron, Antibiotics, Adolescents, Burkina Faso

\section{Background}

Most genital tract commensals and pathogens require iron for growth and adhesion, including Gardnerella vaginalis [1], Trichomonas vaginalis [2] and Candida albicans [3]. In contrast, most Lactobacillus species, which maintain a healthy vaginal ecosystem, do not require an iron substrate [4]. There have been no previous randomised trials assessing whether vaginal infections are influenced by host iron status. Local iron is available from secreted lactoferrin [5], transudated ferritin and transferrin [6, 7], and menstrual haem iron. Host iron status could affect growth and adhesion of bacterial vaginosis (BV) [8], which is associated with a massive overgrowth of vaginal organisms. Dysbiosis enhances human immunodeficiency viral infectivity [9] and increases by two-fold the risk of preterm birth and perinatal death [10]. Higher concentrations of transferrin in cervico-vaginal fluid were correlated with preterm birth risk in a previous study, suggesting that iron availability must be considered [11].

In areas where anaemia is common, the World Health Organization (WHO) recommends routine iron supplementation [12]. It is currently unknown whether this affects bacterial profiles in the reproductive tract, especially in already iron replete women. In routine programmes, these women receive additional supplements despite there being good evidence that elevated iron stores predispose to systemic infection and inflammation [13]. However, data are absent regarding non-malarial infection risk following iron supplementation during pregnancy [14], or following intermittent supplementation in menstruating women [15]. In the context of a double blind, randomised, controlled, noninferiority trial to assess safety of periconceptional iron supplementation in relation to malaria among young women in Burkina Faso [16], we assessed whether weekly iron supplementation, or baseline host iron status, affected markers of genital infection. The trial design provided two cohorts, namely a large nulliparous cohort who remained nonpregnant and received up to 18 months supplementation, and a primigravid cohort who conceived during the trial.

\section{Methods}

The trial protocol and amendments were approved by ethical review boards and regulatory authorities at each collaborating centre. This sub-study was conducted within a randomised trial of the safety of weekly iron and folic acid supplementation in young women exposed to malaria. The primary specified outcome was BV prevalence, whereas the secondary outcomes were nonBV Nugent scores, vaginal discharge and $\mathrm{pH}>4.5$. Finally, exploratory outcomes were microbiota and drug prescriptions (antibiotics, antifungals, analgesics). Additional data on the main trial and other details relevant to this paper are provided in Additional files 1, 2, 3 and 4.

\section{General procedures}

Between April 2011 and January 2014 a randomised, double blind, controlled trial was conducted in rural Burkina Faso in the Nanoro Health and Demographic Surveillance System area [17], situated $85 \mathrm{~km}$ from Ouagadougou (Additional file 1). Two previous surveys in Burkina Faso indicated a BV prevalence of approximately $6-8 \%[18,19]$. HIV prevalence is low and reported as $1.2 \%$ among women aged $15-49$ years and $0.76 \%$ among pregnant women [20]. Women were recruited from 30 villages and individual and guardian written consents were obtained. Healthy nulliparous, non-pregnant women aged 15-24 years received either weekly ferrous gluconate and folic acid (intervention), or folic acid alone (control) as a directly observed therapy. Participation continued for 18 months for women who did not conceive or, if they became pregnant, they entered the pregnant cohort. Weekly supplementation continued until a scheduled first antenatal visit (ANC1), which was the trial primary end-point.

\section{Non-pregnant cohort}

At enrolment demographic data and medical/obstetric histories were recorded and women were clinically examined. Height (nearest mm), weight (nearest $100 \mathrm{~g}$ ), and mid-upper arm circumference (mm; MUAC) were measured in duplicate. A venous blood sample $(5 \mathrm{~mL})$ was collected for later iron biomarker assessments. Two self-taken swabs were requested for a BV slide and $\mathrm{pH}$ measurement. Samples were not collected during menses. Women symptomatic for $T$. vaginalis and BV were 
treated with single dose metronidazole ( 2 g orally), and those symptomatic for $C$. albicans with miconazole (200 mg intravaginally, daily for 3 days). All participants received a single dose of albendazole (400 $\mathrm{mg}$ ) and praziquantel (1500-2400 mg according to height).

Participants were individually randomised to receive either a capsule containing ferrous gluconate $(60 \mathrm{mg})$ and folic acid $(2.8 \mathrm{mg})$, or an identical capsule containing folic acid alone $(2.8 \mathrm{mg})$, as then recommended by WHO [15]. This regimen was directly observed at weekly visits and continued for up to 18 months, when an end assessment (FIN) was completed and repeat vaginal swabs for BV and vaginal $\mathrm{pH}$ were requested. At FIN, duplicate swabs were additionally requested for preparation of vaginal fluid eluates for microbiota and $T$. vaginalis PCR assays. Swabs were kept cool until returned to the laboratory within 2-4 hours. A venous blood sample $(5 \mathrm{~mL})$ was obtained for iron biomarkers and malaria microscopy.

Women were monitored for pregnancy at weekly visits and symptoms of illness were recorded by a field worker. Symptomatic participants were directed to attend a health centre for free medical treatment for participant care, as this was required for a safety trial. Health centre staff recorded all visits, and a medically qualified researcher collated information on symptoms, clinical diagnoses and treatments. Pregnant women were instructed to attend Nanoro hospital for ANC1.

\section{Pregnant cohort}

At ANC1 (primary endpoint), scheduled at 13-16 weeks' gestation, a venous blood sample $(5 \mathrm{~mL})$ was obtained for iron biomarkers and malaria microscopy, and selftaken vaginal swabs requested as for the non-pregnant cohort. An ultrasound examination was completed to date gestational age. Symptomatic women were treated for BV and T. vaginalis with metronidazole $500 \mathrm{mg}$ orally twice daily for 7 days, for C. albicans with intravaginal miconazole $200 \mathrm{mg}$ daily for 3 days, and for $N$. gonorrhoeae and C. trachomatis with ceftriaxone $250 \mathrm{mg}$ intramuscularly once and amoxycillin $500 \mathrm{mg}$ orally thrice daily for 7 days for suspected cervical infection. Women were encouraged to deliver at their closest health centre. The study provided free obstetric care.

\section{Laboratory procedures \\ Bacterial vaginosis}

BV slides were fixed, Gram stained, air dried and forwarded to the Microbiology Laboratories, Central Manchester University Hospital NHS Trust, UK, for Nugent scoring [21]. Approximately $10 \%$ of slides, plus indeterminate slides, were read in duplicate. Nugent scores of 7-10 indicated BV, 4-6 intermediate, and 0-3 normal flora. Gram stains were available for $80.5 \%$ of women at enrolment, $76.5 \%$ at FIN, and $94.6 \%$ at $\mathrm{ANC1}$, with no differences between trial arms.

\section{Vaginal eluates}

Each tube containing a swab for vaginal eluate was processed on laboratory arrival when $5 \mathrm{~mL}$ of PBS was added to the tube and shaken at high speed (5 minutes), before pipetting and freezing $\left(-20{ }^{\circ} \mathrm{C}\right)$. Vaginal eluates were air freighted on dry ice to the University of Northumbria, UK.

\section{Malaria microscopy}

Blood films were stained with Giemsa and read independently by two qualified microscopists and, in the case of discordant results, by a third reader.

\section{Iron status}

At the Nanoro Research Laboratories, ferritin, indicative of iron stores, and serum transferrin receptor (sTfR), indicative of functional iron deficit, were measured in duplicate by ELISA (Spectro Ferritin S-22 and TFC 94 Transferrin Receptor, RAMCO Laboratories Inc., Texas) and C-reactive protein (CRP) by ELISA (EU59131, IBL International, GMBH, Hamburg). Definitions of iron deficiency were (1) adjusted ferritin (adjFE) allowing for inflammation, ferritin $<15 \mu \mathrm{g} / \mathrm{L}$ if $\mathrm{CRP}<10 \mu \mathrm{g} / \mathrm{mL}$, or ferritin $<70 \mu \mathrm{g} / \mathrm{L}$ if $\mathrm{CRP} \geq 10 \mu \mathrm{g} / \mathrm{mL}$; or (2) a ratio of sTfR $\mu \mathrm{g} / \mathrm{mL}$ to $\log _{10}$ ferritin $>5.6$, [22], which assesses both stored and functional iron and is possibly less affected by inflammation.

\section{Microbiota/T. vaginalis $q P C R$}

DNA was extracted from vaginal eluates using the PowerLyzer Power Soil kit (MoBio, SD, USA) with the following modifications. A $250-\mu \mathrm{L}$ aliquot of vaginal eluate was combined with $500 \mu \mathrm{L}$ of bead solution added to the bead tube and processed following the manufacturer's instructions. DNA extraction negative controls were processed for each kit and sequenced alongside test samples. Bacterial profiling of the variable region 4 (V4) of the $16 \mathrm{~S}$ rRNA gene was performed by NU-OMICS (Northumbria University) based on the Schloss wet-lab MiSeq procedure [23], and T. vaginalis by qPCR [24] (Additional file 2).

\section{Statistical analysis}

The sample size was determined from formal power calculations for the malaria trial endpoints [25]. Analyses excluded women who remained non-menarcheal throughout the trial and any who became menarcheal within 6 months of FIN when menses may have been irregular. The primary analyses were comparisons of BV (Nugent $>6$ ) risk at ANC1 and at FIN (menarcheal) by arm on an intention to treat basis using a binomial model with a log link, and baseline infection and use of 
antibiotics in the 3 months prior to assessment as covariates. Other infection markers were analysed similarly. Nugent scoring gave a 3-level classification and each level was compared to the remaining levels. Intention to treat analyses of iron deficiency used a similar approach with no covariates.

Associations between baseline infections and dichotomised iron deficiency markers were similarly analysed, but with no covariate for antibiotic use, as free study prescriptions had not commenced. The analysis adjusted for MUAC, a surrogate for nutritional status. Associations between baseline infection indicators and continuous biomarkers followed similar binomial models, with log-transformed values scaled by their standard deviations to give comparable risk ratios across markers. The $P$ values for multiple markers were adjusted for multiple testing using the false discovery rate method [26].

Receipt of antibiotics, antifungals and analgesics was classified by clinical indication. Poisson regression models were used to estimate incidence ratios and to test for differences between arms, using number of visits between enrolment and assessment as an exposure time offset. Incidence rates are presented per 50 visits as this approximated the mean number of visits prior to followup assessment. For separate indications, $P$ values were adjusted using the false discovery rate approach.

The statistical methods for analysis and visualisation of microbiota communities are described in Additional file 2. Multinomial regression models were used to identify if Community State Type (CST) was associated with trial arm, pregnancy, iron deficiency or infection markers.

\section{Results \\ Participants}

A total of 1959 nulliparous women were randomised and 1954 were included in the intention to treat dataset (Fig. 1). During follow-up, 478 (24.5\%) women became pregnant, with 1476 remaining non-pregnant; 315 (65.9\%) were assessed at ANC1, with two-thirds of these visits occurring at $<20$ weeks' gestation. Among the non-pregnant cohort, defined as menarcheal and followed up to 18 months, 877 (56.6\%) women completed an end assessment survey (FIN). Women lost to follow-up differed in some baseline characteristics (Additional file 3: Table S1). Table 1 shows baseline participant characteristics, categorised by subsequent status in constituting the pregnant or non-pregnant cohorts. Trial arms were well balanced. In the intention to treat analysis, the median (interquartile range) number of directly observed treatments as a percentage of the number of weeks in the study was $79 \%(58-90 \%, \mathrm{n}=1954)$, with no difference by trial arm. For the total sample, adolescents ( $<20$ years) comprised $93 \%$. BV prevalence was $12.3 \%$, with $8.9 \%$ having intermediate flora. BV correlated with MUAC (RR per $\mathrm{cm} \mathrm{1.23;} \mathrm{95 \%} \mathrm{CI} \mathrm{1.08-1.41,} P=0.003$ ) and BMI (RR per $\mathrm{kg} / \mathrm{m}^{2} 1.23$; 95\% CI 1.08-1.40, $P=$ $0.002)$, but not with age $(P=0.82)$. Prevalence of vaginal

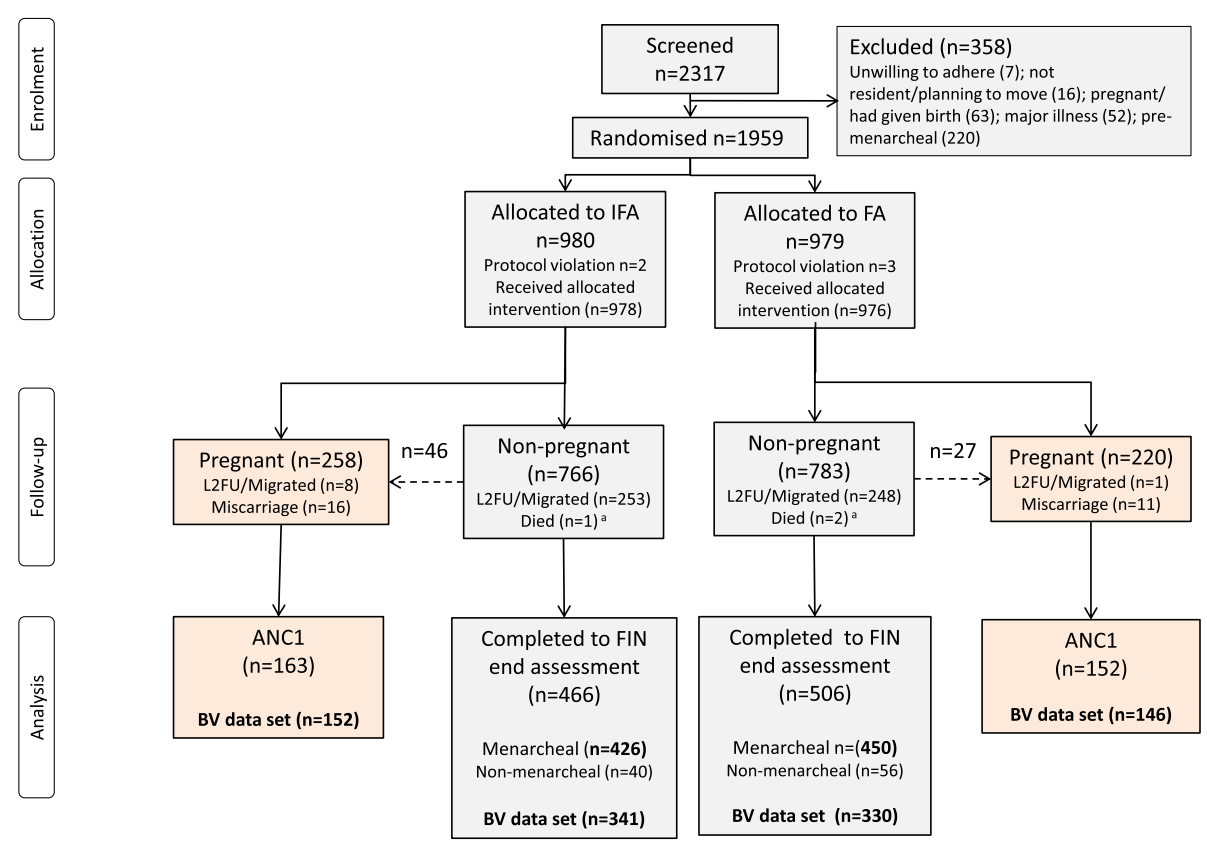

a Three deaths: one accidental drowning, one probable lymphosarcoma, one undiagnosed chest pain

Fig. 1 Participant flow chart 
Table 1 Baseline characteristics by trial arm of participants who subsequently became pregnant or remained non-pregnant during follow-up

\begin{tabular}{|c|c|c|c|c|c|c|}
\hline \multirow[t]{3}{*}{ Variable } & \multicolumn{3}{|c|}{ Pregnant cohort } & \multicolumn{3}{|c|}{ Non-pregnant cohort } \\
\hline & \multirow[t]{2}{*}{$\mathrm{N}^{\mathrm{a}}$} & \multirow{2}{*}{$\begin{array}{l}\text { Iron } \\
N=258\end{array}$} & \multirow{2}{*}{$\begin{array}{l}\text { Control } \\
N=220\end{array}$} & \multirow[t]{2}{*}{$\mathrm{N}^{\mathrm{a}}$} & \multirow{2}{*}{$\begin{array}{l}\text { Iron } \\
N=766\end{array}$} & \multirow{2}{*}{$\begin{array}{l}\text { Control } \\
N=783\end{array}$} \\
\hline & & & & & & \\
\hline \multicolumn{7}{|l|}{ Sociodemographic } \\
\hline Age, years, mean (SD) & 478 & $17.1(1.7)$ & $17.1(1.7)$ & 1549 & $16.7(1.7)$ & $16.7(1.8)$ \\
\hline \multicolumn{7}{|l|}{ Ethnic group, n (\%) } \\
\hline Mossi & 478 & $250(96.9)$ & $214(97.3)$ & 1548 & $730(95.3)$ & $757(96.8)$ \\
\hline Other & 478 & $8(3.1)$ & $6(2.7)$ & 1548 & $36(4.7)$ & $25(3.2)$ \\
\hline \multicolumn{7}{|l|}{ Marital status } \\
\hline Married & 478 & $22(8.5)$ & $15(6.8)$ & 1544 & $17(2.2)$ & $18(2.3)$ \\
\hline Never married & 478 & $236(91.5)$ & $204(92.7)$ & 1544 & $748(97.8)$ & $760(97.6)$ \\
\hline Previously married & 478 & $0(0)$ & $1(0.5)$ & 1544 & $0(0)$ & $1(0.1)$ \\
\hline \multicolumn{7}{|l|}{ Occupation, ${ }^{b}$ n (\%) } \\
\hline Student & 478 & $60(23.3)$ & $51(23.2)$ & 1549 & $255(33.3)$ & $274(35.0)$ \\
\hline Trading & 478 & $10(3.9)$ & $10(4.5)$ & 1549 & $24(3.1)$ & $21(2.7)$ \\
\hline Domestic labour & 478 & $154(59.7)$ & $120(54.5)$ & 1549 & $401(52.3)$ & $407(52.0)$ \\
\hline Farming & 478 & $130(50.4)$ & $101(45.9)$ & 1549 & $269(35.1)$ & $290(37.0)$ \\
\hline Other & 478 & $3(1.2)$ & $3(1.4)$ & 1549 & $3(0.4)$ & $2(0.3)$ \\
\hline \multicolumn{7}{|l|}{ Education, n (\%) } \\
\hline No schooling & 477 & $176(68.5)$ & $142(64.5)$ & 1544 & $447(58.6)$ & $450(57.6)$ \\
\hline Primary & 478 & $54(20.9)$ & $38(17.3)$ & 1549 & $168(21.9)$ & $169(21.6)$ \\
\hline Lower secondary & 477 & $24(9.3)$ & $38(17.3)$ & 1544 & $144(18.9)$ & $153(19.6)$ \\
\hline Higher secondary & 477 & $3(1.2)$ & $2(0.9)$ & 1544 & $4(0.5)$ & $9(1.2)$ \\
\hline Literate, $\mathrm{n}(\%)$ & 473 & $68(26.7)$ & $62(28.4)$ & 1530 & $278(36.8)$ & $297(38.4)$ \\
\hline \multicolumn{7}{|l|}{ Reproductive history and infections, n (\%) } \\
\hline Menarcheal & 478 & $241(93.4)$ & $203(92.3)$ & 1549 & $639(83.4)$ & $645(82.4)$ \\
\hline Menarche age, mean (SD) & 443 & $14.9(1.0)$ & $14.8(1.1)$ & 1279 & $15(1.0)$ & $15.1(1.1)$ \\
\hline Ever had sex & 478 & $98(38)$ & $82(37.3)$ & 1548 & $159(20.8)$ & $164(21.0)$ \\
\hline Uses contraception & 478 & $54(20.9)$ & $51(23.2)$ & 1549 & $106(13.8)$ & $86(11.0)$ \\
\hline Uses condoms & 478 & $53(20.5)$ & $50(22.7)$ & 1549 & $103(13.4)$ & $81(10.3)$ \\
\hline Nugent 7-10 & 391 & $33(15.7)$ & $23(12.7)$ & 1247 & $80(13.0)$ & $68(10.8)$ \\
\hline Nugent 4-6 & 391 & $17(8.1)$ & $11(6.1)$ & 1247 & $49(8.0)$ & $66(10.5)$ \\
\hline Nugent $0-3$ & 391 & $160(76.2)$ & $147(81.2)$ & 1247 & $487(79.1)$ & $497(78.8)$ \\
\hline Vaginal discharge & 478 & $3(1.2)$ & $4(1.8)$ & 1549 & $13(1.7)$ & $12(1.5)$ \\
\hline Vaginal $\mathrm{pH} \geq 4.5$ & 394 & $119(55.9)$ & $84(46.4)$ & 1280 & $317(50.3)$ & $328(50.5)$ \\
\hline \multicolumn{7}{|l|}{ Nutrition and iron biomarkers, $\mathrm{n}(\%)$} \\
\hline Drinks alcohol & 476 & $140(54.5)$ & $119(54.3)$ & 1549 & $372(48.6)$ & $380(48.5)$ \\
\hline $\mathrm{BMI}, \mathrm{kg} / \mathrm{m}^{2}$, mean (SD) & 478 & $20.2(1.8)$ & $20.3(2.1)$ & 1549 & $19.7(2.2)$ & $19.6(2.2)$ \\
\hline MUAC, cm, mean (SD) & 478 & $24.1(1.8)$ & $24.3(2.2)$ & 1549 & $23.6(2.1)$ & $23.6(2.2)$ \\
\hline Iron deficient (adjFE) & 467 & $25(10.0)$ & $26(12.0)$ & 1528 & $84(11.1)$ & $100(13.0)$ \\
\hline Iron deficient (ratio sTfR/log ferritin) & 470 & $52(20.6)$ & $55(25.3)$ & 1524 & $162(21.4)$ & $168(21.9)$ \\
\hline \multicolumn{7}{|l|}{ Adherence to treatment } \\
\hline $\mathrm{DOT}^{\mathrm{C}}$ mean (SD) & 478 & $70.3(27.0)$ & $71.4(26.0)$ & 1549 & $70.0(27.8)$ & $70.8(26.3)$ \\
\hline
\end{tabular}

${ }^{\mathrm{a}}$ Total responders

${ }^{b}$ More than one response allowed

${ }^{c}$ Number of directly observed treatments as a percentage of the number of weeks from enrolment to assessment

adjFE adjusted ferritin, $B M I$ body mass index, DOT directly observed treatment, MUAC mid-upper arm circumference, SD standard deviation, sTfR serum

transferrin receptor 
discharge was $1.6 \%$. Vaginal $\mathrm{pH} \geq 4.5$ was recorded in $50.6 \%$, with no difference between arms.

\section{Infections and iron status at baseline assessment}

Baseline prevalence of iron deficiency was lower using the single iron biomarker adjFE than when using the combined marker sTfR/log ferritin, but with either marker, it did not differ by trial arm (Table 1). Prevalence of iron deficiency remained very similar by trial arm using varying CRP cut-offs $(5-25 \mu \mathrm{g} / \mathrm{mL}$ ) (Additional file $4 \mathrm{~b}$ ). Iron replete participants at baseline were less likely to have normal vaginal flora (Nugent score 0 3) (adjFE: $P=0.036$; sTfR $/ \log$ ferritin ratio: $P=0.255$ ). Vaginal discharge was reported by only a few women but was less prevalent with iron repletion (adjFE: $P=$ 0.03 ; sTfR $/ \log$ ferritin ratio: $P=0.06$ ) (Table 2). A sensitivity analysis using alternative lower ferritin thresholds of $<15 \mu \mathrm{g} / \mathrm{L}$ and $\mathrm{CRP}<10 \mu \mathrm{g} / \mathrm{mL}$, or $<30 \mu \mathrm{g} / \mathrm{L}$ and $\mathrm{CRP} \geq 10 \mu \mathrm{g} / \mathrm{mL}$, for iron depletion adjusting for inflammation was explored to assess whether a lower ferritin cut-off significantly altered these associations (Additional file 3: Table S2). Prevalence of vaginal discharge remained significantly lower in iron replete women $(P=$ $0.013)$, and normal vaginal flora remained significantly less frequent in iron replete women $(P=0.028)$. High $\mathrm{pH}$ was positively associated with plasma ferritin (adjFE) $(P$ $=0.015$ adjusted for MUAC), but not the sTfR/log ferritin ratio $(P=0.14)$ (Additional file 3: Table S3).

\section{Lower genital tract infection in the pregnant cohort ANC1}

Prevalence of iron deficiency (adjFE) did not differ significantly between trial arms (6.9\% (iron) and 12.7\% (control); RR 0.54, 95\% CI 0.27-1.1, P=0.12) or based on the sTfR/log ferritin ratio (11.1\% (iron) and $12.7 \%$ (control); RR 0.88, 95\% CI 0.48-1.61, $P=0.73$ ).

Prevalence was similar in iron and control arms (Table 3) for BV (7.9\% vs. 6.2\%; RR 0.93, 95\% CI 0.332.56, $P=0.88)$, intermediate flora $(11.2 \%$ vs. $13.0 \% ; P=$ $0.86), T$. vaginalis $(15.6 \%$ vs. $10.1 \% ; P=0.17)$, and vaginal discharge ( $8.6 \%$ vs. $7.9 \% ; P=0.85)$. Abnormal discharge occurred in $24 \%$ of women with BV and $8 \%$ of those with $T$. vaginalis.

\section{Lower genital tract infection in the non-pregnant cohort FIN}

Prevalence of iron deficiency (adjFE) was 9.0\% (iron) and $11.0 \%$ (control) (RR 0.82, 95\% CI 0.55-1.22, $P=0.37$ ) and, based on sTfR/log ferritin ratio, it was $20.1 \%$ (iron) and 21.2\% (control) (RR 0.95, 95\% CI 0.73-1.23, $P=$ $0.74)$.

No prevalence difference was observed for BV (13.5\% iron vs. $12.1 \%$ control, RR $0.86,95 \%$ CI $0.56-1.34, P=$ $0.51)$, intermediate flora $(10.3 \%$ vs. $7.9 \%, P=0.47)$, or $T$. vaginalis $(5.6 \%$ vs. $4.2 \%, P=0.43)$ (Table 3$)$. Vaginal discharge was more frequent in iron-supplemented women (11.5\% vs. $6.9 \%, P=0.026)$. This difference would be

Table 2 Baseline host iron status using two definitions of iron deficiency and infection markers ( $\mathrm{n}=1673$, excluding 281 nonmenarcheal)

\begin{tabular}{|c|c|c|c|c|c|c|c|c|}
\hline \multirow[t]{2}{*}{ Infection } & \multirow[t]{2}{*}{$\mathrm{N}$} & \multirow[t]{2}{*}{ Replete (\%) } & \multirow[t]{2}{*}{ Deficient (\%) } & \multicolumn{2}{|l|}{ Unadjusted } & \multicolumn{3}{|c|}{ Adjusted $^{a}$} \\
\hline & & & & RR (95\% Cl) & $P$ value ${ }^{b}$ & $\overline{\mathrm{N}^{c}}$ & $\mathrm{RR}(95 \% \mathrm{Cl})$ & $P$ value \\
\hline \multicolumn{9}{|l|}{ Adjusted ferritin } \\
\hline$B V^{d}$ & 1347 & & & & 0.127 & 1326 & & 0.032 \\
\hline Nugent $7-10^{\mathrm{e}}$ & 1347 & 142/1151 (12.3) & 15/175 (8.6) & $1.44(0.87-2.39)$ & 0.168 & 1326 & $1.53(0.92-2.54)$ & 0.104 \\
\hline Nugent $4-6^{e}$ & 1347 & 108/1151 (9.4) & $11 / 175$ (6.3) & $1.49(0.82-2.72)$ & 0.203 & 1326 & $1.47(0.81-2.68)$ & 0.210 \\
\hline Nugent $0-3^{e}$ & 1347 & $901 / 1151$ (78.3) & 149/175 (85.1) & $0.92(0.86-0.99)$ & 0.036 & 1326 & $0.92(0.86-0.98)$ & 0.016 \\
\hline Vaginal discharge & 1673 & $21 / 1430(1.5)$ & $8 / 214(3.7)$ & $0.39(0.18-0.88)$ & 0.044 & 1644 & $0.41(0.18-0.92)$ & 0.030 \\
\hline $\mathrm{pH} \geq 4.5$ & 1371 & $608 / 1166(52.1)$ & 86/183 (47.0) & $1.11(0.94-1.31)$ & 0.204 & 1349 & $1.11(0.94-1.30)$ & 0.222 \\
\hline \multicolumn{9}{|c|}{ sTfR/log ferritin ratio > 5.6} \\
\hline$B V^{d}$ & 1347 & & & & 0.513 & 1324 & & 0.224 \\
\hline Nugent $7-10^{e}$ & 1347 & $127 / 1030$ (12.3) & $30 / 294(10.2)$ & $1.21(0.83-1.76)$ & 0.358 & 1324 & $1.25(0.86-1.83)$ & 0.239 \\
\hline Nugent $4-6^{e}$ & 1347 & $95 / 1030(9.2)$ & 24/294 (8.2) & $1.13(0.74-1.73)$ & 0.644 & 1324 & $1.12(0.73-1.71)$ & 0.619 \\
\hline Nugent $0-3^{e}$ & 1347 & $808 / 1030$ (78.4) & 240/294 (81.6) & $0.96(0.90-1.02)$ & 0.255 & 1324 & $0.96(0.90-1.02)$ & 0.231 \\
\hline Vaginal discharge & 1673 & $18 / 1270(1.4)$ & $11 / 372(3.0)$ & $0.48(0.23-1.01)$ & 0.070 & 1642 & $0.49(0.23-1.04)$ & 0.062 \\
\hline $\mathrm{pH} \geq 4.5$ & 1371 & $551 / 1044(52.8)$ & $144 / 303(47.5)$ & $1.11(0.97-1.27)$ & 0.117 & 1347 & $1.11(0.97-1.26)$ & 0.126 \\
\hline
\end{tabular}

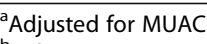

${ }^{\mathrm{b}}$ Fishers Exact Test

${ }^{\mathrm{c}}$ Number of observations in adjusted analysis

${ }^{\mathrm{d}}$ Global test (Fishers/ordinal regression) for BV as 3-category outcome

eEach group compared to both other groups

$B V$ bacterial vaginosis, sTfR serum transferrin receptor 
Table 3 Lower genital tract infection indicators in pregnant (ANC1) and non-pregnant (FIN) cohorts by trial arm

\begin{tabular}{|c|c|c|c|c|c|c|c|c|c|}
\hline \multirow[t]{2}{*}{ Assessment } & \multirow[t]{2}{*}{ Infection marker } & \multirow[t]{2}{*}{$\mathrm{N}$} & \multicolumn{2}{|l|}{ Trial arm } & \multicolumn{2}{|l|}{ Unadjusted } & \multicolumn{3}{|c|}{ Adjusted $^{\mathrm{a}}$} \\
\hline & & & Iron (\%) & Control (\%) & RR $(95 \% \mathrm{Cl})$ & $P$ value $^{b}$ & $\overline{\mathrm{N}^{c}}$ & RR $(95 \% \mathrm{Cl})$ & $P$ value ${ }^{c}$ \\
\hline Pregnant & Bacterial vaginosis $^{d}$ & 298 & & & & 0.776 & 245 & & 0.704 \\
\hline ANC1 & Nugent $7-10^{e}$ & 298 & 12/152 (7.9) & $9 / 146(6.2)$ & $1.28(0.56-2.95)$ & 0.653 & 245 & $0.93(0.33-2.56)$ & 0.881 \\
\hline \multirow[t]{5}{*}{$N=315$} & Nugent $4-6^{e}$ & 298 & 17/152 (11.2) & 19/146 (13.0) & $0.86(0.47-1.59)$ & 0.723 & 245 & $0.94(0.47-1.88)$ & 0.858 \\
\hline & Nugent $0-3^{e}$ & 298 & 123/152 (80.9) & $118 / 146(80.8)$ & $1.00(0.90-1.12)$ & 1 & 245 & $1.03(0.91-1.15)$ & 0.665 \\
\hline & T. vaginalis ${ }^{f}$ & 286 & 23/147 (15.6) & $14 / 139(10.1)$ & $1.55(0.83-2.90)$ & 0.217 & 286 & $1.55(0.83-2.90)$ & 0.168 \\
\hline & Vaginal discharge & 315 & 14/163 (8.6) & 12/152 (7.9) & $1.09(0.52-2.28)$ & 0.841 & 315 & $1.08(0.51-2.25)$ & 0.846 \\
\hline & $\mathrm{pH} \geq 4.5$ & 309 & $92 / 158(58.2)$ & $86 / 151(57.0)$ & $1.02(0.84-1.24)$ & 0.908 & 260 & $1.06(0.86-1.31)$ & 0.555 \\
\hline Non-pregnant & Bacterial vaginosis $^{\mathrm{d}}$ & 671 & & & & 0.588 & 546 & & 0.676 \\
\hline $\mathrm{FIN}^{f}$ & Nugent $7-10^{e}$ & 671 & 46/341 (13.5) & $40 / 330(12.1)$ & $1.11(0.75-1.65)$ & 0.645 & 546 & $0.86(0.56-1.34)$ & 0.509 \\
\hline \multirow[t]{4}{*}{$N=877$} & Nugent $4-6^{e}$ & 671 & $35 / 341(10.3)$ & 26/330 (7.9) & $1.30(0.80-2.11)$ & 0.347 & 546 & $1.21(0.72-2.03)$ & 0.467 \\
\hline & Nugent $0-3^{e}$ & 671 & 260/341 (76.2) & $264 / 330(80.0)$ & $0.95(0.88-1.03)$ & 0.263 & 546 & $0.99(0.90-1.08)$ & 0.806 \\
\hline & T. vaginalis ${ }^{f}$ & 716 & 20/360 (5.6) & $15 / 356(4.2)$ & $1.32(0.69-2.53)$ & 0.489 & 716 & $1.30(0.67-2.50)$ & 0.432 \\
\hline & Vaginal discharge & 874 & 49/425 (11.5) & $31 / 449(6.9)$ & $1.67(1.09-2.57)$ & 0.019 & 874 & $1.63(1.06-2.51)$ & 0.026 \\
\hline
\end{tabular}

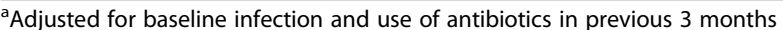

${ }^{\mathrm{b}}$ Fishers exact test

'Logistic regression

${ }^{d}$ Each group compared to both other groups

Excludes those less than 6 months post menarcheal

faseline not available

considered non-significant allowing for the number of secondary outcomes tested. Abnormal discharge occurred in $9 \%$ of those with BV and $18 \%$ with $T$. vaginalis.

\section{Comparison of non-pregnant and pregnant cohorts} Cross-sectional BV prevalence was higher in nonpregnant women at FIN (12.8\%) than at ANC1 (7.0\%) (RR 1.8, 95\% CI 1.2-2.9, $P=0.011$, adjusted for antibiotic use), but $T$. vaginalis prevalence was lower (4.9\%) (RR $0.38,95 \%$ CI $0.24-0.59, P<0.001)$. P. falciparum prevalence was $54 \%$ at $\mathrm{ANC} 1$ and $41 \%$ at FIN, despite free access to anti-malarial treatments.

\section{Antibiotic courses}

The number of antibiotic courses (excluding antimalarials) provided to the non-pregnant cohort was higher in women receiving iron supplements $(P=0.014)$ (Table 4). These also received more anti-fungal treatments $(P=0.014)$ and analgesics $(P=0.008)$. BV and $T$. vaginalis were mostly asymptomatic and, as these infections were unlikely to account for the high proportion of women receiving antibiotics, all indications for antibiotic prescription at Health Centres were assessed (Table 5). The most frequent indications were respiratory illness, gastrointestinal, reproductive tract and local infections. Gastrointestinal infections were treated more frequently in iron-supplemented women $(P=0.005$, allowing for the multiple indications tested) (Table 5). With far fewer events, no significant differences were detected in the pregnant cohort at ANC1 (Additional file 3: Table S4).

\section{Vaginal microbiota}

Figure 2 demonstrates the distribution of the top 20 taxa for all samples combined by trial arm, study visit and BV infection. Three CSTs typified this population, corresponding to CST I (40.4\%), III (24.6\%) and IV (35\%), dominated by Lactobacillus crispatus, Lactobacillus iners and a mixed community with reduced lactobacilli, respectively. CST IV was more frequent in the nonpregnant cohort at FIN than pregnant women at ANC1 $(P<0.001)$. CST IV was associated with BV $(P<0.001)$ and $T$. vaginalis $(P<0.001)$ (Table 6$)$. No differences were observed in CST IV frequency or alpha- and betadiversity, by trial arm, or host iron status, in pregnant or non-pregnant cohorts (Additional file 4c). Iron replete women at ANC1 and FIN had non-significantly higher Shannon diversity. CST frequencies did not differ significantly from earlier results using the lower ferritin threshold (Additional file 3: Table S5).

\section{Discussion}

In this young population, reported sexual activity was low at recruitment but its commencement carried a substantial risk of immediate pregnancy, a sexually transmitted infection ( $T$. vaginalis), an unstable microbiota and malaria, which was more frequent in primigravidae. Evidence on effects of intermittent iron supplementation on infectious disease outcomes is scarce and unclear, 
Table 4 Health Centre treatment courses prescribed to pregnant and non-pregnant cohorts by trial arm from enrolment to ANC1 (pregnant) or FIN (non-pregnant)

\begin{tabular}{|c|c|c|c|c|c|c|c|c|}
\hline \multirow[t]{2}{*}{ Treatment } & \multicolumn{2}{|c|}{ Number of treatments } & \multicolumn{2}{|c|}{ Mean treatments per person } & \multicolumn{2}{|c|}{ Mean treatments per 50 visits $^{a}$} & \multirow[t]{2}{*}{ Incidence ratio ${ }^{\mathrm{b}}(95 \% \mathrm{Cl})$} & \multirow[t]{2}{*}{$P$ value } \\
\hline & Iron & Control & Iron & Control & Iron & Control & & \\
\hline Pregnant & & & $n=163$ & $n=152$ & Visits $=6907$ & Visits $=6235$ & & \\
\hline Antibiotics & 92 & 73 & 0.564 & 0.480 & 0.666 & 0.585 & $1.14(0.84-1.55)$ & 0.411 \\
\hline Anti-fungals ${ }^{c}$ & 16 & 15 & 0.098 & 0.099 & 0.116 & 0.120 & $0.96(0.47-1.95)$ & 0.916 \\
\hline Analgesics & 107 & 107 & 0.656 & 0.704 & 0.775 & 0.858 & $0.90(0.69-1.18)$ & 0.454 \\
\hline Non-pregnant & & & $n=426$ & $n=451$ & Visits $=26473$ & Visits $=27633$ & & \\
\hline Antibiotics & 331 & 284 & 0.777 & 0.630 & 0.625 & 0.514 & $1.22(1.04-1.43)$ & 0.015 \\
\hline Anti-fungals ${ }^{c}$ & 39 & 21 & 0.092 & 0.047 & 0.074 & 0.038 & $1.94(1.14-3.30)$ & 0.014 \\
\hline Analgesics & 481 & 421 & 1.129 & 0.933 & 0.908 & 0.762 & $1.19(1.05-1.36)$ & 0.008 \\
\hline
\end{tabular}

${ }^{\mathrm{a}}$ Mean number of visits: 42 in pregnant and 63 in non-pregnant

$\mathrm{b}$ Incidence ratio was computed using Poisson regression with the number of visits as the exposure period

${ }^{c}$ More than $90 \%$ of vaginal pessaries prescribed for genital tract infections

with few studies and small sample sizes [15]. This randomised, controlled, double blind trial found no evidence that $60 \mathrm{mg}$ iron $/ 2.8 \mathrm{mg}$ folic acid supplements offered weekly, increased the risk of BV, T. vaginalis, or CST IV microbiota in either the pregnant or non-pregnant cohort. A two-fold increased use of antibiotics for treatment of gastrointestinal infections, with increased use of antifungals for lower genital infection in the non-pregnant iron supplemented cohort, raises new concerns about the safety of weekly iron supplements for young women. $\mathrm{Nu}$ tritional status, reflected by MUAC, was an effect modifier for BV infection. This result adds to a growing body of evidence of an association between diet and BV, for which there is currently no clear explanation [27].

A major strength of this study was its large cohort of non-pregnant peri-menarcheal adolescents, as well as a concurrent pregnancy cohort [16]. Some loss to followup occurred due to movement outside the study area following marriage, unwanted pregnancy or, if unmarried, working for relatives living elsewhere. Trial participants were young, nulliparous girls, with low prevalence of iron deficiency at recruitment. Accurate estimation of

Table 5 Mean number of health centre antibiotic treatments in non-pregnant cohort by infection diagnosis and trial arm from enrolment to FIN

\begin{tabular}{|c|c|c|c|c|c|c|c|}
\hline Infection indication & Iron & Control & $\begin{array}{l}\text { Iron per } 50 \text { visits } \\
n=26,473\end{array}$ & $\begin{array}{l}\text { Control per } 50 \text { visits } \\
n=27,633\end{array}$ & $\begin{array}{l}\text { Incidence ratio }{ }^{\mathrm{a}} \\
(95 \% \mathrm{Cl})\end{array}$ & $P$ value & $\mathrm{PFDR}^{\mathrm{b}}$ \\
\hline$\overline{\text { Malaria }^{c}}$ & 41 & 28 & 0.077 & 0.051 & $1.53(0.94-2.47)$ & 0.084 & 0.278 \\
\hline Respiratory & 75 & 87 & 0.142 & 0.157 & $0.90(0.66-1.23)$ & 0.503 & 0.719 \\
\hline Local $^{d}$ & 75 & 57 & 0.142 & 0.103 & $1.37(0.97-1.94)$ & 0.071 & 0.278 \\
\hline Gastrointestinal $^{e}$ & 62 & 30 & 0.117 & 0.054 & $2.16(1.39-3.34)$ & $<0.001$ & 0.005 \\
\hline Urinary tract ${ }^{f}$ & 10 & 9 & 0.019 & 0.016 & $1.16(0.47-2.86)$ & 0.747 & 0.934 \\
\hline $\mathrm{STI} \mathrm{I}^{\mathrm{g}}$ & 13 & 7 & 0.025 & 0.013 & $1.94(0.77-4.86)$ & 0.158 & 0.292 \\
\hline Genital $^{h}$ & 15 & 16 & 0.028 & 0.029 & $0.98(0.48-1.98)$ & 0.952 & 0.993 \\
\hline Dental & 11 & 5 & 0.021 & 0.009 & $2.30(0.80-6.62)$ & 0.123 & 0.292 \\
\hline Meningitis & 0 & 3 & 0 & 0.005 & $0(N A)$ & 0.993 & 0.993 \\
\hline Miscellaneous ${ }^{i}$ & 29 & 42 & 0.055 & 0.076 & $0.72(0.45-1.16)$ & 0.175 & 0.292 \\
\hline
\end{tabular}

Incidence ratio was computed using Poisson regression with the number of visits as the exposure period

${ }^{\mathrm{b}} P$ value adjusted for multiple testing using false discovery rate method [26]

${ }^{c}$ Antibiotics in addition to anti-malarial treatment

${ }^{\mathrm{d}}$ Non-enteric and non-respiratory, includes ear, skin, ophthalmic, abscess, wounds, local trauma, sinusitis, mastoiditis, keloid, scalp ringworm

e'Dysentry, typhoid, enteritis, intestinal parasitosis, gastric ulcer, gastroenteritis, diarrhoea and abdominal pain, vomiting and abdominal pain, amoebiasis, colopathy, sub-occlusion

${ }^{\mathrm{f}}$ Cystitis, dysuria

Includes syphilis, trichomoniasis

hupper or lower genital infection

'Itching, colic, headache, generalised or localised pain, fever, vomiting only, anaemia, anxiety, neuralgia, spasms, urticarial, plus non-infectious specific diagnoses: cancers, fertility problems, angina, self-medication, allergy, burns, renal stones, contraception, thyroid and cardiac diseases, epilepsy, filariasis, foreign body, venomous bites, fractures, migraine, HIV, varicella, mumps, prophylactic and non-classifiable: includes three uncertain descriptors, or absent statement PFDR positive false discovery rate, STI sexually transmitted infection 


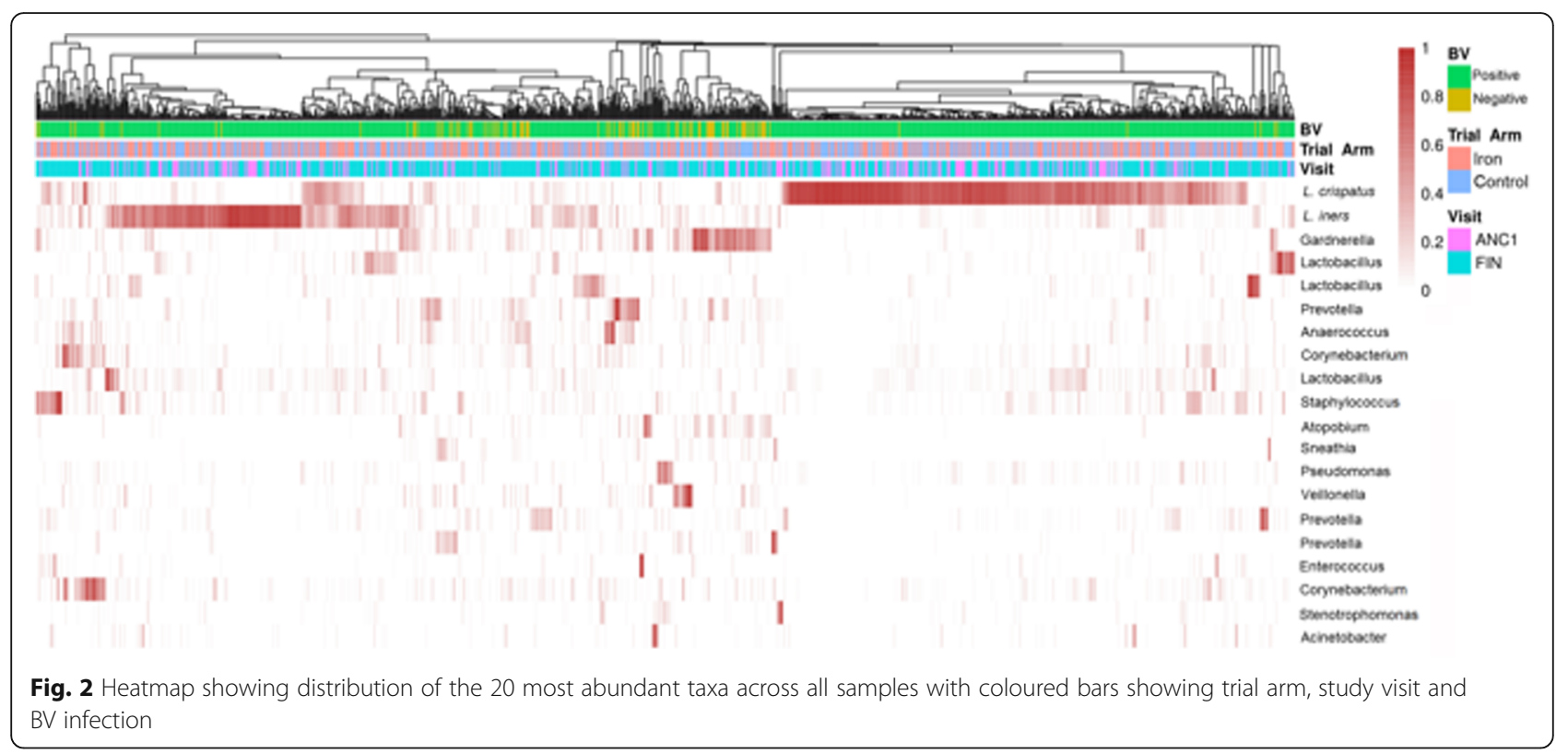

iron deficiency prevalence is influenced by effects of inflammation on indices such as ferritin. A recent meta-analysis in women of reproductive age experiencing inflammation estimated the difference of depleted iron stores between adjusted and unadjusted ferritin values as $2-8$ median percentage points, dependent on the adjustment method [28]. We used a high ferritin cut-off of $<70 \mu \mathrm{g} / \mathrm{L}$ for iron deficiency in women with $\mathrm{CRP} \geq 10 \mu \mathrm{g} / \mathrm{mL}$, allowing for inflammation, as well as the lower cut-off $<15 \mu \mathrm{g} / \mathrm{L}$ with $\mathrm{CRP}<10 \mu \mathrm{g} / \mathrm{L}$ to include those without inflammation. CRP concentration increases in early pregnancy, which was accommodated by using the $\mathrm{CRP}<10 \mu \mathrm{g} / \mathrm{L}$ cut-off. Although prevalence of iron deficiency in absolute terms varied according to the CRP cut-off used, sensitivity analyses showed that trends and trial arm differences in iron deficiency were similar irrespective of CRP level. We additionally used the $\mathrm{sTfR} / \log _{10}$ ferritin ratio $>5.6$, which is derived from an intermediate ferritin cut-off of $<30 \mu \mathrm{g} / \mathrm{L}$ [22]. We considered this provided a reasonable estimate of iron deficiency. Sensitivity analysis of alternative ferritin thresholds for defining iron deficiency, adjusting for inflammation, gave very similar results.

Despite good supplementation adherence over an 18 month period, facilitated by weekly direct observation, there was no significant improvement in systemic iron biomarkers. The absence of an association between iron supplementation and BV or T. vaginalis prevalence could relate to the lack of intervention efficacy in reducing iron deficiency. At baseline, iron-replete participants were less likely to have normal vaginal flora, which suggests host iron status is reflected in the mucosa. Following supplementation, an increased prescription for fungal infections was also observed inferring an iron- fungal infection interaction [29]. Antibiotics used for treatment of enteric infections may have altered lower genital tract bacterial profiles. Cessation of menses with pregnancy would also reduce iron availability to haemutilising pathogens.

Chronic inflammation from malaria, which was frequent, as well as other infections, would increase hepcidin release, restricting iron absorption. In Beninese women with afebrile $P$. falciparum parasitaemia, dietary iron absorption was reduced by approximately $40 \%$ with infection [30]. In the non-pregnant cohort, a significantly higher number of antibiotic-treated gastrointestinal infections - predominantly diagnosed as dysentery, typhoid, and intestinal parasitosis - occurred in supplemented women, possibly related to unabsorbed iron becoming available to colonic gut microbiota [30]. Although some misclassification of infection categories would arise due to a lack of laboratory confirmation, this should not alter findings by trial arm. Increased gut inflammation and pathogenicity following iron supplementation, or fortification, is reported in children exposed to high infection pressure in Ghana [31] and Kenya [32], and increased risk of bloody dysentery and respiratory infection in Pakistan [33]. This is the first report in young women to show increased enteric symptoms requiring antibiotic treatments following iron supplementation. Concurrent infection with non-typhi salmonella and $P$. falciparum should be considered [34]. Increased use of antifungal prescriptions in the iron-supplemented nonpregnant cohort is of interest because, both in the gut and vaginal mucosa, C. albicans is a normal commensal, competing with other organisms for nutrients, and increased fungal virulence due to iron malabsorption could be 
Table 6 Community state type distribution in pregnant and non-pregnant (menarcheal) cohorts by trial arm, iron deficiency and infection status

\begin{tabular}{|c|c|c|c|c|c|c|c|}
\hline Variable & Visit & Group & $n$ & $\begin{array}{l}\text { CST I } \\
\text { n (\%) }\end{array}$ & $\begin{array}{l}\text { CST III } \\
\text { n (\%) }\end{array}$ & $\begin{array}{l}\text { CST IV } \\
\text { n (\%) }\end{array}$ & $P$ value \\
\hline \multirow[t]{4}{*}{ Trial arm } & \multirow[t]{2}{*}{ ANC1 } & Iron & 144 & $70(48.6)$ & $39(27.1)$ & $35(24.3)$ & \multirow[t]{2}{*}{0.518} \\
\hline & & Control & 136 & $61(44.9)$ & $45(33.1)$ & $30(22.1)$ & \\
\hline & \multirow[t]{2}{*}{$\mathrm{FIN}^{\mathrm{b}}$} & Iron & 349 & $121(34.7)$ & $88(25.2)$ & $140(40.1)$ & \multirow[t]{2}{*}{0.113} \\
\hline & & Control & 341 & $140(1.1)$ & $66(19.4)$ & $135(9.6)$ & \\
\hline \multirow[t]{4}{*}{ Iron deficiency, Adjusted ferritin } & \multirow[t]{2}{*}{ ANC1 } & No & 249 & $118(47.4)$ & $75(30.1)$ & $56(22.5)$ & \multirow[t]{2}{*}{0.57} \\
\hline & & Yes & 27 & $10(37.0)$ & $9(33.3)$ & $8(29.6)$ & \\
\hline & \multirow[t]{2}{*}{$\mathrm{FIN}^{\mathrm{b}}$} & No & 620 & $237(38.2)$ & $140(22.6)$ & $243(39.2)$ & \multirow[t]{2}{*}{0.281} \\
\hline & & Yes & 65 & $20(30.8)$ & $13(20.0)$ & $32(49.2)$ & \\
\hline \multirow[t]{4}{*}{ Ratio sTfR/log ferritin } & \multirow[t]{2}{*}{ ANC1 } & No & 247 & $120(48.6)$ & $74(30.0)$ & $53(21.5)$ & \multirow[t]{2}{*}{0.216} \\
\hline & & Yes & 32 & $11(34.4)$ & $10(31.2)$ & $11(34.4)$ & \\
\hline & \multirow[t]{2}{*}{$\mathrm{FIN}^{\mathrm{b}}$} & No & 544 & $205(37.7)$ & $124(22.8)$ & 215 (39.5) & \multirow[t]{2}{*}{0.81} \\
\hline & & Yes & 140 & $52(37.1)$ & $29(20.7)$ & $59(42.1)$ & \\
\hline \multirow[t]{6}{*}{ Nugent score ${ }^{c}$} & \multirow[t]{3}{*}{ ANC1 } & $7-10$ & 15 & $0(0)$ & $1(6.7)$ & $14(93.3)$ & $<0.001$ \\
\hline & & $4-6$ & 31 & $7(22.6)$ & $6(19.4)$ & $18(58.1)$ & $<0.001$ \\
\hline & & $0-3$ & 221 & $116(52.5)$ & 74 (33.5) & $31(14.0)$ & $<0.001$ \\
\hline & \multirow[t]{3}{*}{$\mathrm{FIN}^{\mathrm{b}}$} & $7-10$ & 80 & $2(2.5)$ & $6(7.5)$ & $72(90.0)$ & $<0.001$ \\
\hline & & $4-6$ & 60 & $11(18.3)$ & $6(10.0)$ & $43(71.7)$ & $<0.001$ \\
\hline & & $0-3$ & 501 & $232(46.3)$ & $132(26.3)$ & $137(27.3)$ & $<0.001$ \\
\hline \multirow[t]{4}{*}{ T. vaginalis } & \multirow[t]{2}{*}{ ANC1 } & No & 243 & $121(49.8)$ & $73(30.0)$ & $49(20.2)$ & \multirow[t]{2}{*}{0.006} \\
\hline & & Yes & 37 & $10(27.0)$ & $11(29.7)$ & $16(43.2)$ & \\
\hline & \multirow[t]{2}{*}{$\mathrm{FIN}^{\mathrm{b}}$} & No & 655 & $260(39.7)$ & $149(22.7)$ & $246(37.6)$ & \multirow[t]{2}{*}{$<0.001$} \\
\hline & & Yes & 35 & $1(2.9)$ & $5(14.3)$ & $29(82.9)$ & \\
\hline \multirow[t]{2}{*}{ High $\mathrm{pH} \geq 4.5$} & \multirow[t]{2}{*}{ ANC1 } & No & 123 & $73(59.3)$ & $37(30.1)$ & $13(10.6)$ & \multirow[t]{2}{*}{$<0.001$} \\
\hline & & Yes & 153 & $58(37.9)$ & $45(29.4)$ & $50(32.7)$ & \\
\hline \multirow[t]{4}{*}{ Vaginal discharge } & \multirow[t]{2}{*}{ ANC1 } & No & 260 & $122(46.9)$ & $80(30.8)$ & $58(22.3)$ & \multirow[t]{2}{*}{0.268} \\
\hline & & Yes & 20 & $9(4.5)$ & $4(20.0)$ & $7(35.0)$ & \\
\hline & $\mathrm{FIN}^{\mathrm{b}}$ & No & 618 & $232(37.5)$ & $144(23.3)$ & $242(39.2)$ & 0.205 \\
\hline & & Yes & 69 & $28(40.6)$ & $10(14.5)$ & $31(44.9)$ & \\
\hline Antibiotics within previous three months & ANC1 & No & 252 & $118(46.8)$ & $73(29.0)$ & $61(24.2)$ & NA \\
\hline & & Yes & 28 & $13(46.4)$ & $11(39.3)$ & $4(14.3)$ & \\
\hline & $\mathrm{FIN}^{\mathrm{b}}$ & No & 616 & $236(38.3)$ & $136(22.1)$ & $244(39.6)$ & NA \\
\hline & & Yes & 74 & $25(33.8)$ & $18(24.3)$ & $31(41.9)$ & \\
\hline
\end{tabular}

${ }^{a} P$ value for association between status and CST from a multinomial regression model adjusting for antibiotic use in the 3 months prior to assessment

${ }^{\mathrm{b}}$ Menarcheal women only

${ }^{\mathrm{C}}$ Each Nugent group compared to the other two. BV corresponds to Nugent score 7-10

ANC1 first antenatal visit, CST community state type, FIN end assessment, sTfR serum transferrin receptor

anticipated. In addition, disruption to normal bacterial gut populations due to tetracycline treatment can result in candida overgrowth, reducing colonisation resistance [35]. The intestinal tract is considered as a reservoir of infection leading to recurrent vaginal candidiasis based on the correspondence between vaginal and stool samples [36].

Self-taken vaginal swabs proved acceptable, providing samples for gram stains, vaginal eluates and microbiota assays. The absence of differences in microbiota CST categories by trial arm is consistent with no supplementation effect on BV or T. vaginalis. BV was associated with CST IV, and both had lower prevalence in pregnant than non-pregnant women [37]. This pregnancy difference is probably related to hormonal factors [38], although we cannot exclude the possibility that it is mediated by differences in other characteristics between those who became pregnant and those that did not. In marked contrast, $T$. vaginalis prevalence, also associated 
with CST IV, was three-fold higher at ANC1 compared to non-pregnant women at FIN, probably due to more regular sexual activity with male partners untreated for this sexually transmitted infection. Interactions between BV and T. vaginalis are poorly understood [39] but, under experimental conditions, T. vaginalis is associated with reduced populations of Lactobacillus spp, but not BV spp [40]. Co-infection in early pregnancy may hinder transition to stable microbiome communities, such as CST I, which is associated with better pregnancy outcomes [41]. Clinical outcomes may depend on initial host iron status, as experimental studies have shown altered inflammatory responses, higher abundance of Bifidobacteriaceae and Lactobacillaceae, and prolonged intestinal nematode survival in animals receiving irondeficient diets [42].

At baseline, elevated vaginal $\mathrm{pH}$ values $(\geq 4.5)$ were observed in iron-replete participants and were positively associated with plasma ferritin $(P=0.015)$. High vaginal $\mathrm{pH}$ may be attributable to normal pubertal changes [43], young age, irregular menses [44], and factors impacting on lactobacilli or other lactic acid producing microbes [45], but also to genital tract infection. The reason in this study for the significant association of high $\mathrm{pH}$ and more disturbed flora in adolescents with better iron stores before supplementation is unknown and warrants further investigation.

\section{Conclusions}

There was no evidence that weekly iron increased the risk of BV or T. vaginalis infections, but the intervention was considered poorly absorbed as systemic iron biomarkers were not significantly changed. Iron supplementation did result in more gastrointestinal morbidity, leading to increased antibiotic prescription and genital tract infection requiring antifungal treatments in the non-pregnant cohort. This is a concern since recently revised WHO recommendations are shifting in favour of providing intermittent daily iron [46]. A daily regime could be more likely to exacerbate gut microbes and/or increase antibiotic/antifungal prescriptions. Since malaria is the most likely cause of iron malabsorption in the gut, iron supplementation studies in malaria endemic areas need to profile enteric and vaginal infections, as well as antibiotic use.

\section{Additional files}

Additional file 1: Background data to the main RCT. (DOCX $18 \mathrm{~kb}$ )

Additional file 2: Detailed methods for microbiota and T. vaginalis $\mathrm{qPCR}$ profiling. (DOCX $13 \mathrm{~kb}$ )

Additional file 3: Tables not shown in Results. (DOCX 49 kb)
Additional file 4: (a) Legend to Additional figures; (b) CRP sensitivity analysis and iron deficiency; (c) Figure Shannon Diversity (microbiota results). (ZIP $116 \mathrm{~kb})$

\section{Acknowledgements}

We gratefully acknowledge the contribution and support of participating women, local communities, the memory of his late Majesty Naaba Tigré of Nanoro, study teams, female field assistants, and nurses, midwives, supervisors, doctors and staff of Nanoro Health District and peripheral Health Centres, St Camille hospital. Additionally, we thank the laboratory assistance of Greg Harper and Marc Tahita; quality control staff at G\&G Food Supplies Ltd, East Grinstead, West Sussex, UK; members of the Data Safety and Monitoring Board; Chris Roberts, Chair, University of Manchester, UK; Patrick van Rheenen, University of Groningen, Netherlands; Marleen Boelaert and Veerle Van Lerberghe, Institute of Tropical Medicine, Antwerp, Belgium; and Professor Stephen Cummings, Associate Academic Dean Health and Life Sciences, who facilitated microbiota analyses at Northumbria University. We are grateful to Raffaella Ravinetto and Céline Schurmans of the Clinical Trials Unit, Institute of Tropical Medicine, Antwerp, and Isidore Traore for their efforts on trial monitoring activities.

\section{Funding}

This work was supported by the National Institutes of Health (Grant Number U01HD061234-01A1; Supplementary -05S1 and -02S2), the National Institute of Child Health and Human Development, and the National Institutes of Health Office of Dietary Supplements. The grant covered the cost of all field work, including local salaries, and funded the Tropical Institute in Belgium for the salary costs of SG, who was stationed in Burkina Faso. The University of Manchester received part-time salary costs for statistical analysis by SR; JB received pro rata costs for slide reading. The University of Northumbria was given a sub-contract for microbiota profiling. Salary costs of LB, BJB and YC were borne by their own academic institutions. The Scientific Advisory Committee was reimbursed for travelling expenses.

The contents of this publication are solely the responsibility of the authors and do not necessarily represent the views of the funding organisation, which played no role in data analysis.

\section{Availability of data and materials}

The trial protocol was reported in the Lancet (www.thelancet.com/protocolreviews/10PRT-6932). Until placed in a public repository, data relating to the current study can be requested from the corresponding author and will be made available following an end-user data agreement and sponsor approval.

\section{Authors' contributions}

BB was the Principal Investigator for the main RCT on iron supplementation and is accountable for all aspects of the work; he co-wrote the paper. LB conceived the study and wrote the paper. SR was co-author and study statistician. SG was the clinical and field co-ordinator for the research. Iron assays were conducted in Burkina Faso by SD, microbiome by AN and CS at Northumbria University, UK, and BV gram stains by JB at Manchester, UK. AZ, $\mathrm{SO}$ and $\mathrm{YC}$ were responsible for data management and safety monitoring. BF was the main trial statistician. UDA and HT were members of the Scientific

Advisory Committee and reviewed the paper. All authors read and approved the final manuscript

\section{Ethics approval and consent to participate}

The clinical protocol was approved by the Liverpool School of Tropical Medicine, UK, Research Ethics Committee (LSTM/REC), the Institutional Review Board of the Institute of Tropical Medicine, Antwerp, Belgium (IRB/ ITM), the Antwerp University Hospital Ethics Committee (EC/UZA), the Institutional Ethics Committee of Centre Muraz (Comité d'Ethique Institutionnel du Centre Muraz, CEI/CM), and the National Ethics Committee (Comité Ethique pour la Recherche en Santé, CERS) in Burkina Faso. Prior to enrolment, the study team visited each village to inform village elders and senior women about trial objectives and for permission to invite young women to take part. Informed consent with right to withdraw (signature or thumb print) was granted by each participant, or by her appointed guardian if a minor or married at recruitment, and repeated later by participants continuing to be followed in the pregnant cohort. 


\section{Consent for publication}

Participants provided written informed consent for publication of research results.

\section{Competing interests}

The authors declare that they have no competing interests.

\section{Publisher's Note}

Springer Nature remains neutral with regard to jurisdictional claims in published maps and institutional affiliations.

\section{Author details}

${ }^{1}$ Division of Cancer Sciences, Faculty of Biology, Medicine and Health, School of Medical Sciences, University of Manchester, Manchester Academic Health Science Centre, Manchester, UK. ${ }^{2}$ Centre for Biostatistics, Division of Population Health, Health Services Research and Primary Care, Faculty of Biology, Medicine and Health, School of Medical Sciences, University of Manchester, Manchester Academic Health Science Centre, Manchester, UK. ${ }^{3}$ Department of Biomedical Sciences, Prince Leopold Institute of Tropical Medicine, Antwerp, Belgium. ${ }^{4}$ Medical Mission Institute, Würzburg, Germany. ${ }^{5}$ Faculty of Health and Life Sciences, Northumberland Building, University of Northumbria, Newcastle-upon-Tyne, England. ${ }^{6}$ Clinical Research Unit, Institute for Research in Health Sciences, (IRSS-URCN), Nanoro, Burkina Faso. ${ }^{7}$ Molecular Virology and Microbiology, Baylor College of Medicine, Houston Texas, USA. ${ }^{8}$ Central Manchester University Hospitals NHS Foundation Trust, Microbiology Department, Manchester, UK. ${ }^{9}$ Clinical Trials Unit, Prince Leopold Institute of Tropical Medicine, Antwerp, Belgium. ${ }^{10}$ Medical Research Council Unit (MRC), Banjul, Gambia. "'London School of Hygiene and Tropical Medicine, London, UK. ${ }^{12}$ Clinical Division, Liverpool School of Tropical Medicine, Liverpool, UK. ${ }^{13}$ Liverpool School of Tropical Medicine and Institute of Infection and Global Health, University of Liverpool, Liverpool, UK. ${ }^{14}$ Global Child Health Group, Academic Medical Centre, University of Amsterdam, Amsterdam, The Netherlands. ${ }^{15}$ Division of Cancer Sciences, 5th (Research) floor, St Mary's Hospital, Oxford Road, Manchester M13 9WL, UK.

Received: 26 April 2017 Accepted: 27 October 2017

Published online: 23 November 2017

\section{References}

1. Jarosik GP. Identification of a Gardnerella vaginalis haemoglobin-binding protein. Curr Microbiol. 2001;42:49-52.

2. Arroyo R, Cárdenas-Guerra RE, Figueroa-Angulo EE, Puente-Rivera J, Zamudio-Prieto O, Ortega-LÓpez J. Trichomonas vaginalis cysteine proteinases: iron response in gene expression and proteolytic activity. Biomed Res Int. 2015;2015:946787.

3. Almeida RS, Wilson D, Hube B. Candida albicans iron acquisition within the host. FEMS Yeast Res. 2009;9:1000-12.

4. Macklaim JM, Gloor GB, Anukam KC, Cribby S, Reid G. At the crossroads of vaginal health and disease, the genome sequence of Lactobacillus iners AB1. Proc Natl Acad Sci U S A. 2011;108:4688-95.

5. Baker EN, Baker HM, Kidd RD. Lactoferrin and transferrin: functional variations on a common structural framework. Biochem Cell Biol. 2002;80:27-34.

6. Tang L-J, De Seta F, Odreman F, et al. Proteomic analysis of human cervicalvaginal fluids. J Prot Res. 2007;6:2874-83.

7. Sriyosachati S, Cox CD. Siderophore-mediated iron acquisition from transferrin by Pseudomonas aeruginosa. Infect Immun. 1986;52:885-91.

8. Singh PK, Parsek MR, Greenberg EP, Welsh MJ. A component of innate immunity prevents bacteria biofilm development. Nature. 2002;417:552-5.

9. Zilberman-Schapira G, Zmora N, Itav S, Bashiardes S, Elinav H, Elinav E. The gut microbiome in human immunodeficiency virus infection. BMC Med. 2016;14:83. doi:10.1186/s12916-016-0625.

10. Brabin L, Brabin BJ, Gies S. Influence of iron status on risk of maternal or neonatal infection and on neonatal mortality with an emphasis on developing countries. Nutr Rev. 2013;71:528-40.

11. Pereira $L$, Reddy AP, Jacob $T$, et al. Identification of novel protein biomarkers of preterm birth in human cervical-vaginal fluid. J Prot Res. 2007;6:1269-76.

12. World Health Organization. Guideline: Intermittent Iron and Folic Acid Supplementation in Menstruating Women. Geneva: WHO; 2011.

13. Wessling-Resnick M. Iron homeostasis and the inflammatory response. Annu Rev Nutr. 2010;30:105-22.
14. Peña-Rosas JP, De-Regil LM, Garcia-Casal MN, Dowswell T. Daily oral iron supplementation during pregnancy. Cochrane Database Syst Rev. 2015;7: CD004736.

15. Fernández-Gaxiola AC, De-Regil LM. Intermittent iron supplementation for reducing anaemia and its associated impairments in menstruating women. Cochrane Database Syst Rev. 2011;12:CD009218. doi:10.1002/14651858. CD009218.pub2.

16. Brabin BJ, Gies $S$, Owens $S$, et al. Perspectives on the design and methodology of periconceptional nutrient supplementation trials. Trials. 2016;17:58.

17. Derra K, Rouamba E, Kazienga A, et al. Profile: Nanoro health and demographic surveillance System. Int J Epidemiol. 2012;41:1293-301.

18. Kirakoya-Samadoulougou F, Nagot N, Defer MC, Yaro S, Meda N, Robert A Bacterial vaginosis among pregnant women in Burkina Faso. Sex Transm Dis. 2008:35:985-89.

19. Kirakoya-Samadoulougou F, Nagot N, Defer MC, et al. Epidemiology of herpes simplex virus type 2 infection in rural and urban Burkina Faso. Sex Transm Dis. 2011;38:117-23.

20. Institut National de la Statistique et de la Démographie (INSD) et ICF International. Enquète Demographique et de Santé et Indicateurs Multiples du Burkina Faso 2010. Calverton, MA: INSD and ICF International; 2012.

21. Nugent RP, Krohn MA, Hillier SI. Reliability of diagnosing bacterial vaginosis is improved by a standardized method of gram stain interpretation. J Clin Microbiol. 1991;29:297-301.

22. Phiri KS, Calis JC, Siyasiya A, Bates I, Brabin B, van Hensbroek MB. New cutoff values for ferritin and soluble transferrin receptor for the assessment of iron deficiency in children in a high infection pressure area. J Clin Pathol. 2009;62:1103-06.

23. Kozich JJ, Westcott SL, Baxter NT, Highlander SK, Schloss PD. Development of a dual-index sequencing strategy and cultivation pipeline for analyzing amplicon sequence data in the MiSeq Illumina sequencing platform. Appl Environ Microbiol. 2013;79(17):5112-20.

24. Caliendo AM, Jordan JA, Green AM, Ingersoll J, Diclemente R, Wingood GM. Real-time PCR improves detection of Trichomonas vaginalis infection compared with culture using self-collected vaginal swabs. Infect Dis Obstet Gynecol. 2005;13:145-50.

25. The Lancet. Protocol summaries. Protocol 10PRT/6932: Malaria risk prior to and during early pregnancy in nulliparous women receiving long-term weekly iron and folic acid supplementation (WIFS): a non-inferiority randomised controlled trial [NCT 01210040]. http://www.thelancet.com/ protocol-reviews/10PRT-6932. Accessed July 2017.

26. Benjamini $Y$, Hochberg $Y$. Controlling the false discovery rate: a practical and powerful approach to multiple testing. J Royal Stats Soc Series. 1995; B57:289-300.

27. Thoma ME, Klebanoff MA, Rovner AJ, et al. Bacterial vaginosis is associated with variation in dietary indices. J Nutr. 2011;141:1698-704.

28. Namaste SM, Rohner F, Huang J, et al. Adjusting ferritin concentrations for inflammation: Biomarkers Reflecting Inflammation and Nutritional Determinants of Anemia (BRINDA) project. Am J Clin Nutr. 2017;106:359S-71S.

29. Bairwa $G$, Jung WH, Kronstad JW. Iron acquisition in fungal pathogens of humans. Metallomics. 2017;9:215-27.

30. Cercamondi Cl, Egli IM, Ahouandjinou E, et al. A febrile Plasmodium falciparum parasitemia decreases absorption of fortification iron but does not affect systemic iron utilization: a double stable-isotope study in young Beninese women. Am J Clin Nutr. 2010;92:1385-92.

31. Zlotkin S, Newton S, Aimone AM, et al. Effect of iron fortification on malaria incidence in infants and young children in Ghana: a randomized trial. JAMA. 2013;310:938-47.

32. Jaeggi $T$, Kortman $G A$, Moretti $D$, et al. Iron fortification adversely affects the gut microbiome, increases pathogen abundance and induces intestinal inflammation in Kenyan infants. Gut. 2015;64:731-42.

33. Soofi S, Cousens S, lqbal SP, et al. Effect of provision of daily zinc and iron with several micronutrients on growth and morbidity among young children in Pakistan: a cluster-randomised trial. Lancet. 2013;382:29-40.

34. Park SE, Pak GD, Aaby P, et al. The relationship between invasive nontyphoidal Salmonella disease, other bacterial bloodstream infections, and malaria in sub-Saharan Africa. Clin Infect Dis. 2016;62 Suppl 1:S23-31.

35. Payne S, Gibson G, Wynne A, Hudspith B, Brostoff J, Tuohy K. In vitro studies on colonization resistance of the human gut microbiota to candida albicans and the effect of tetracycline and Lactobacillus plantarum LPK. Curr Issues Intest Microbiol. 2003;4:1-8. 
36. Miles MR, Olsen L, Rogers A. Recurrent vaginal candidiasis: importance of an intestinal reservoir. JAMA. 1977;238:1836-37.

37. Romero R, Hassan SS, Gajer P, et al. The composition and stability of the vaginal microbiota of normal pregnant women is different from that of non-pregnant women. Microbiome. 2014;2:4-10.

38. Robinson DP, Klein SL. Pregnancy and pregnancy-associated hormones alter immune responses and disease pathogenesis. Horm Behav. 2012;62:263-71.

39. Cauci S, Culhane JF. Modulation of vaginal immune response among pregnant women with bacterial vaginosis by Trichomonas vaginalis, Chlamydia trachomatis, Neisseria gonorrhoeae, and yeast. Am J Obstet Gynecol. 2007;196:133. e1-7.

40. Fichorova RN, Buck OR, Yamamoto HS, et al. The villain team-up or how Trichomonas vaginalis and bacterial vaginosis alter innate immunity in concert. Sex Trans Infect. 2013;89:460-66.

41. Smith SB, Ravel J. The vaginal microbiota, host defence and reproductive physiology. J Physiol. 2016;595(2):451-63. doi:10.1113/JP271694.

42. Kortman GAM, Mulder MLM, Richters TJW, et al. Low dietary intake restrains the intestinal inflammatory response and pathology of enteric infection by food-borne pathogens. Eur J Immunol. 2015;45:2553-67.

43. Thoma ME, Gray RH, Kiwanuka N, Aluma S, Wang M-C, Sewankambo N. Longitudinal changes in vaginal microbiota composition assessed by gram stain among never sexually active pre- and postmenarcheal adolescents in Rakai, Uganda. Pediatr Adolesc Gynecol. 2011;24:42-4.

44. Brabin $L$, Roberts $S A$, Fairbrother $E$, et al. Factors affecting vaginal $p H$ levels among female adolescents attending genitourinary medicine clinics. Sex Trans Infect. 2005:81:483-87.

45. Zhou X, Brown CJ, Abdo Z, et al. Differences in the composition of vaginal microbial communities in healthy Caucasian and black women. ISME J. 2007;1:121-33

46. World Health Organization. Guidelines: Daily Iron Supplementation of Adult Women and Adolescent Girls. Geneva: WHO; 2016.

\section{Submit your next manuscript to BioMed Central and we will help you at every step:}

- We accept pre-submission inquiries

- Our selector tool helps you to find the most relevant journal

- We provide round the clock customer support

- Convenient online submission

- Thorough peer review

- Inclusion in PubMed and all major indexing services

- Maximum visibility for your research

Submit your manuscript at www.biomedcentral.com/submit

) Biomed Central 